Check for updates

Cite this: RSC Adv., 2017, 7, 43994

Received 12th June 2017

Accepted 30th August 2017

DOI: $10.1039 / \mathrm{c} 7 \mathrm{ra06545k}$

rsc.li/rsc-advances

\section{Enhancement of mechanical properties of polymeric nanofibers by controlling crystallization behavior using a simple freezing/thawing process $\uparrow$}

\author{
Hoik Lee, ${ }^{a}$ Kyohei Yamaguchi, ${ }^{a}$ Tomoki Nagaishi, ${ }^{a}$ Masaaki Murai, ${ }^{a}$ \\ Myungwoong Kim, ${ }^{b}$ Kai Wei, ${ }^{c}$ Ke-Qin Zhang (D) *c and Ick Soo Kim (D) *a
}

\begin{abstract}
We demonstrate the modulation of physical and mechanical properties by controlling crystallinity in crosslinked poly(vinyl alcohol) (PVA) nanofibers using a simple and straightforward freezing/thawing process. PVA chains in the cross-linked network are swollen and rearrange through the freezing/thawing process, resulting in the formation of more hydrogen bonding and hence, a higher degree of crystallization in the nanofibers compared to pristine electrospun nanofibers. Quantitative analyses with X-ray diffraction and FT-IR studies confirm increases of the crystallite diameter from $24.2 \AA$ to $28.3 \AA$ and the degree of crystallinity from $23.5 \%$ to $43.6 \%$, respectively. Also, we found that the increase of crystallinity led to a dramatic enhancement of the mechanical properties: the tensile strength was increased up to $165 \%$ compared to pristine nanofibers, while the elongation at break was decreased. This straightforward and facile process will enable us to precisely control crystallinity, and also to fine-tune the physical properties of polymeric nanofibers; consequently, the method will broaden the application of polymeric nanofibers.
\end{abstract}

\section{Introduction}

Crystallinity of polymers has been of interest and has been investigated extensively due to its high impact on intrinsic properties in polymers such as mechanical properties, waterresistance, and gas permeability. ${ }^{1,2}$ Crystallization of polymers is a process associated with chain folding behavior leading to a partial alignment of chains, the so-called semi-crystalline state, which is one of the significant factors affecting physical, thermal and optical properties of polymers..$^{3-5}$ Polymeric nanofibers fabricated by electrospinning have been widely utilized in academia and industry for the past decades due to its high specific surface area, high porosity with excellent pore interconnectivity, and flexibility. ${ }^{6-8}$ Though polymeric nanofibers have attracted significant attention in polymer science and technology, controlling their crystalline structures has not been fully investigated because they typically exhibit amorphous features with a very low degree of crystallinity. ${ }^{9-11}$ The nature of electrospinning process, e.g. fast solvent evaporation rate,

\footnotetext{
${ }^{a}$ Nano Fusion Technology Research Lab, Division of Frontier Fibers, Institute for Fiber Engineering (IFES), Interdisciplinary Cluster for Cutting Edge Research (ICCER), Shinshu University, 3-15-1, Tokida, Ueda, Nagano 386-8567, Japan. E-mail: kim@shinshu-u.ac.jp ${ }^{b}$ Department of Chemistry, Inha University, Incheon 22212, Korea

'National Engineering Laboratory for Modern Silk (NESLab), College of Textile and Clothing Engineering, Soochow University, Suzhou, 215123, China. E-mail: kqzhang@suda.edu.cn

$\dagger$ Electronic supplementary information (ESI) available: Morphological studies of fabricated PVA nanofibers, and FT-IR spectra of pure PVA and cross-linked PVA nanofibers. See DOI: 10.1039/c7ra06545k
}

highly limits to gain opportunities for polymer chains to rearrange and align to crystallize. ${ }^{12,13}$ Therefore, it is important to understand the formation of crystalline structure in nanofibers to further tailor a variety of their properties to achieve target applications.

There have been endeavours to find a route to induce crystallinity in polymer samples. For example, the crystallization of poly(vinyl alcohol) (PVA) has been investigated quite well. ${ }^{14}$ Hassan et al. showed that freezing/thawing PVA hydrogel samples was effective to increase crystallinity of the hydrogel. ${ }^{15}$ Plus, they showed that several parameters, e.g. the number of freezing and thawing cycles, molecular weight of PVA, and the concentration of the solution significantly affected further increasing crystallinity in the polymer matrix. They claimed that an increase in mobility of polymer chain in cross-linked network induced an additional crystallization as the material shrunk and swollen. This concept was further extended to healing process of materials; Zhang et al. reported that physically cross-linked PVA hydrogel treated with freezing/thawing cycles can autonomously be self-healed at room temperature without any needs of stimuli or healing agents. ${ }^{16}$ The treatment of polymer samples with freezing/thawing cycles makes crystalline region in PVA hydrogel larger via rearrangement of PVA chains, allowing more interchain hydrogen bonding between hydroxyl groups in chains. ${ }^{17,18}$ In a practical perspective of the material, PVA is invaluable in a number of applications such as fiber industries due to its excellent chemical resistance, biocompatibility and water-solubility, ${ }^{19}$ therefore, fibrous materials consisting of PVA have been investigated with 
a variety of fabrication methods including an electrospinning technique. $^{20,21}$ It will be beneficial to effectively control its crystallinity in a fibrous structure and hence physical properties, with the process described above for broadening the applicability of PVA fibers.

Herein, we report an effective route to induce the crystallization in PVA nanofibers through post-spinning process, i.e. freezing/thawing method. We fabricated well-defined PVA nanofibers chemically cross-linked with glutaraldehyde via electrospinning and chemical treatment. The crystallinity was largely modulated through freezing/thawing process. Hydrophilic PVA chains was swollen and rearranged to form hydrogen bonding, leading to secondary crystallization in nanofiber structure. In addition, we found that additional freezing/ thawing processes improved mechanical properties, i.e. making nanofiber harder and stiffer. Quantitative parameters, the diameter of crystallite and degree of crystallinity, were extracted from two different experiments: X-ray diffraction (XRD) and Fourier transform infrared spectra (FT-IR), respectively. ${ }^{22,23}$ Careful analyses of the experimental results confirmed increased crystalline size and degree of crystallinity by increasing the number of freezing/thawing cycles. Those two structural parameters were further correlated with physical properties of the nanofibers. The tensile strength was $\sim 165 \%$ increased upon 5 freezing/thawing cycles compared to samples before the process, while elongation at break decreased to lower strain regime. The values of stress and Young's modulus was linearly scaled to both crystallite size and the degree of crystallinity, strongly suggesting that additional crystallization processes led to enhancement of mechanical properties in nanofibers.

\section{Experimental}

\section{Materials}

PVA ( $M_{\mathrm{w}} \sim 104500 \mathrm{~g} \mathrm{~mol}^{-1},>89 \%$ hydrolysis) was purchased from Kuraray Co. Ltd. (Japan). Glutaraldehyde (GA, 50\% in aqueous solution) was obtained from Sigma-Aldrich (USA). Hydrochloric acid ( $\mathrm{HCl}, 37 \%$ in aqueous solution) was purchased from Wako Pure Chemical Industries, Ltd. (Japan). All reagents were used without further purification. Deionized water (DI water) was obtained using Milli-Q system.

\section{Nanofiber preparation}

PVA aqueous solution ( $7 \mathrm{wt} \%$ ) was prepared by vigorously stirring the solution at $80{ }^{\circ} \mathrm{C}$ for $1 \mathrm{~h}$. Upon cooling to room temperature, desired amount of GA (molar ratio of GA to PVA was varied from 30 to 120 moles per PVA mole) was added to find a suitable concentration for freezing/thawing. The prepared solutions were supplied to an electrospinning apparatus through a $5 \mathrm{ml}$ plastic syringe to a capillary tip with an inner diameter of $0.6 \mathrm{~mm}$. A high-voltage power supply (Har100*12, Matsusada Co., Tokyo, Japan), capable of generating voltages up to $100 \mathrm{kV}$, was used as the source of the electric field. A copper wire connected to a positive electrode was attached to an ejection needle, and a negative electrode was linked to a metallic collector. The tip-to-collector distance was $15 \mathrm{~cm}$, and a supplied voltage was $12 \mathrm{kV}$ at $20 \pm 3{ }^{\circ} \mathrm{C}$ with the humidity of $30 \pm 5 \%$ for all electrospinning process. The resultant nanofiber mat was dried under ambient condition to remove residual solvent. To make PVA nanofibers stable and insoluble in water, the resultant electrospun nanofiber mat was exposed to $\mathrm{HCl}$ vapor in a desiccator for $60 \mathrm{s.}^{24}$ In this reaction, GA and $\mathrm{HCl}$ acted as a chemical cross-linking agent and a catalyst, respectively. Cross-linked PVA nanofiber mat was immersed in an aqueous solution and kept at $-20{ }^{\circ} \mathrm{C}$ for $3 \mathrm{~h}$, followed by thawing at $15{ }^{\circ} \mathrm{C}$ for $3 \mathrm{~h}$. This freezing and thawing cycle was repeated up to 5 times to study the effect of freezing/thawing process to crystallinity of nanofiber. Final nanofiber samples were dried at room temperature for $24 \mathrm{~h}$, and then further characterized with a variety of methods.

\section{Characterizations}

The morphologies of prepared nanofibers were studied with scanning electron microscope (SEM, JSM-6010LA, JEOL, Japan). All nanofiber samples were coated with Pt under a fine coater (JEC-1600, JEOL, Japan) before imaging. The average diameter of nanofibers was extracted from SEM images using image analysis software (ImageJ, version 1.49). At least 100 points in each SEM image of the corresponding sample were randomly selected and used to evaluate the average value. To study the crystallinity of prepared nanofiber, wide-angle X-ray diffraction (XRD) experiments were conducted using Mini Flex 300 X-ray diffractometer (Rigaku, Japan). The $\mathrm{Cu} \mathrm{K} \alpha$ radiation was used as X-ray source operating at $50 \mathrm{kV}$ and $200 \mathrm{~mA}$, and diffracted $\mathrm{X}$-ray was detected with an angular range of 5 to $40^{\circ}$ and speed of $5^{\circ} \mathrm{min}^{-1}$. Integrated X-ray powder diffraction software (PDXL ver 2.5, Rigaku Co.) was used for analysis crystallite diameter. Each XRD measurement for crystallite diameter was repeated seven times, and the obtained values were calculated for statistical analysis with excluding maximum and minimum values. Fourier transform infrared (FT-IR) spectroscopic analyses of prepared nanofibers were achieved through a IR Prestige-21, Shimadzu Co. with a resolution of $2 \mathrm{~cm}^{-1}$ by averaging 64 scans in the range $4000-400 \mathrm{~cm}^{-1}$. For a quantitative analysis, baselines were first set to pass through minima in the spectra. Then, the spectra were all normalized with the peak at $1240 \mathrm{~cm}^{-1}$. The degree of crystallinity was calculated using the intensity values at 1095 and $1142 \mathrm{~cm}^{-1}$. The mechanical properties of prepared nanofibers were characterized using universal testing machine (UTM, RTC1250A, A\&D Co., Japan) with a $10 \mathrm{kN}$ load cell at an extension rate of $5 \mathrm{~mm} \mathrm{~min}^{-1}$ and gauge length of $25 \mathrm{~mm}$. The nanofiber sheets were prepared as dog-bone shape with $3 \mathrm{~mm}$ (width) $\times 50 \mathrm{~mm}$ (length). All tests were conducted for seven times to obtain average values of physical parameters with excluding maximum and minimum values for statistical analysis.

\section{Results and discussion}

\section{Morphology and structure of nanofibers}

To optimize electrospinning conditions to fabricate welldefined nanofibers, the concentration of PVA was varied from 
6 to $10 \mathrm{wt} \%$. The morphologies of electrospun nanofibers are presented in Fig. S1. $\uparrow$ The average diameter of nanofibers was increased from $200 \pm 28 \mathrm{~nm}$ at $7 \mathrm{wt} \%$ to $310 \pm 40 \mathrm{~nm}$ at $10 \mathrm{wt} \%$. In lower concentration than $7 \mathrm{wt} \%$, the electrospun nanofibers showed bead morphology with relatively nonuniform diameter. Therefore, we fixed the concentration of $7 \mathrm{wt} \%$ for further studies, where nanofibers were well-defined with relatively uniform and smaller diameter compared to other nanofibers fabricated from PVA solution with the concentration of $6 \mathrm{wt} \%$, $8 \mathrm{wt} \%, 9 \mathrm{wt} \%$, and $10 \mathrm{wt} \%$. Since PVA is soluble in water, its applicability to a range of fields has typically been limited. To address this limit, glutaraldehyde (GA) has been used as a chemical cross-linking agent which can react with the hydroxyl groups of the PVA in the presence of a strong acid. ${ }^{25} \mathrm{~A}$ crosslinked structure can be achieved by reactions between hydroxyl groups in PVA and aldehyde groups in GA in the presence of a strong acid. The reaction mechanism of crosslinking of PVA with GA is presented in Fig. S2. $\dagger$ In the crosslinking reaction, its kinetics is governed by chemical structures and concentrations of polymer and crosslinker, and a concentration of catalyst. We controlled the concentration of crosslinker with fixed polymer concentration and exposure time to acid catalyst. We also varied a molar ratio of GA to PVA from $30: 1$ to $120: 1$ to control a cross-linking density and hence, swelling behavior of crosslinked nanofibers, as presented in Fig. 1. Comparing original nanofibers before swelling to after swelling, it is clearly observed that the morphologies of swollen nanofibers are largely affected by cross-linking density. All cross-linked nanofibers before swelling showed smooth and uniform surface and structure without any beads along the fiber (Fig. S3†). After swelling for 3 days, PVA/GA nanofibers with 1 : 30 molar ratio showed the increase of diameter from $230 \pm$ $27 \mathrm{~nm}$ to $302 \pm 31 \mathrm{~nm}$ with significant morphological change, while the nanofiber sample with 120:1 molar ratio exhibited
Table 1 The changes in the average diameter of the PVA/GA nanofibers as a function of the molar ratio of PVA to GA

\begin{tabular}{lll}
\hline PVA/GA ratio & $\begin{array}{l}\text { Before swelling } \\
(\mathrm{nm})\end{array}$ & $\begin{array}{l}\text { After swelling } \\
\text { (nm) }\end{array}$ \\
\hline $1: 30$ & $230 \pm 27$ & $302 \pm 31$ \\
$1: 60$ & $221 \pm 18$ & $279 \pm 28$ \\
$1: 90$ & $211 \pm 16$ & $248 \pm 21$ \\
$1: 120$ & $198 \pm 12$ & $210 \pm 17$
\end{tabular}

much smaller difference in diameter (from $198 \pm 12 \mathrm{~nm}$ to $210 \pm 17 \mathrm{~nm}$ ) (Table 1). For our purpose, it is desirable for nanofibers to preserve their structure during repeated freezing/ thawing processes; therefore, we fixed the molar ratio of PVA to GA to $1: 120$.

For further proof of crosslinking reactions of PVA and GA, we carried out FT-IR spectroscopy experiments. The FT-IR spectra of unreacted PVA/GA and cross-linked PVA/GA nanofiber samples (120: 1 molar ratio) are presented in Fig. S4. $\dagger$ The both spectra were similar with a broad band at $3000-3600 \mathrm{~cm}^{-1}$, which is assigned to the $\mathrm{O}-\mathrm{H}$ stretching vibrational mode. However, the peak intensity of $\mathrm{O}-\mathrm{H}$ stretching was largely decreased after cross-linking reaction, indicating the loss of hydroxyl group due to the effective reaction of aldehyde group in the crosslinker and hydroxyl group in PVA. Moreover, new absorption peak at $1016 \mathrm{~cm}^{-1}$ has emerged, which is assigned to a characteristic band of the $\mathrm{C}-\mathrm{O}-\mathrm{C}$ in cross-linked PVA, indicating successful crosslinking reactions between hydroxyl groups in PVA and aldehyde groups in GA.

\section{Analysis of crystallite diameter}

$\mathrm{X}$-ray diffraction is one of the most efficient, direct and reliable methods for determining the crystallinity of materials. In order
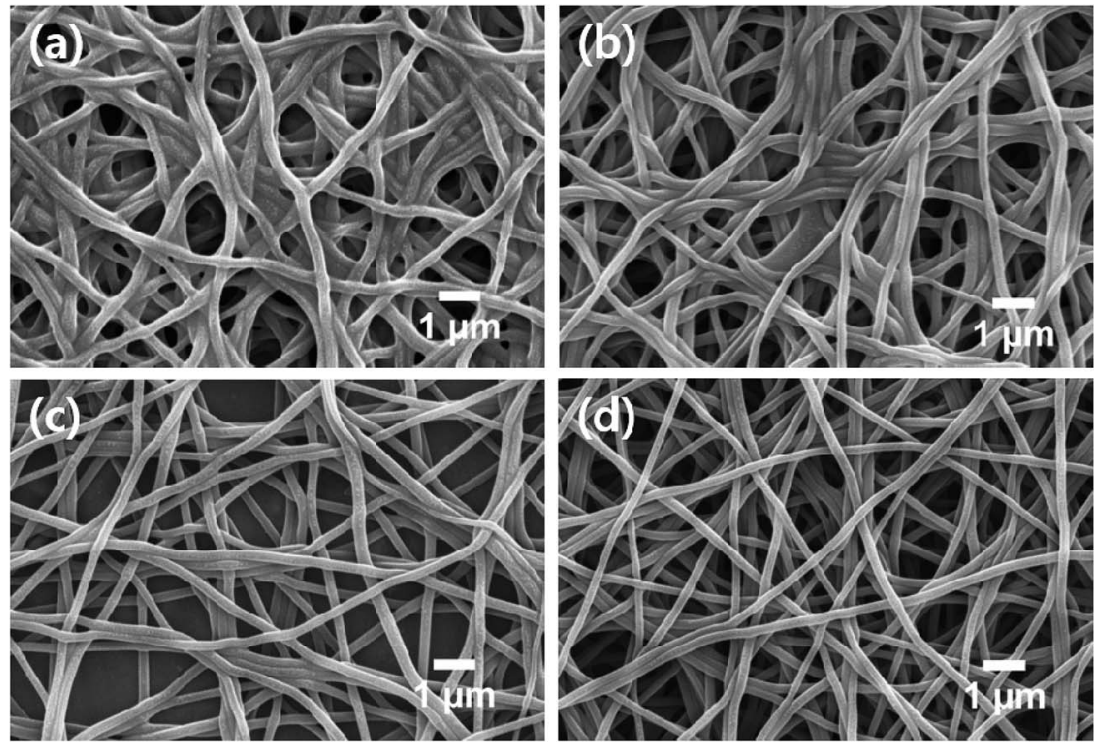

Fig. 1 SEM images showing morphologies of PVA nanofibers prepared with different GA/PVA molar ratio from (a) $30: 1$, (b) $60: 1$, (c) $90: 1$, and (d) $120: 1$ after swelling for 3 days. 
to study the effect of freezing/thawing process to the crystalline structure of nanofibers, we conducted X-ray diffraction experiments for a pure PVA sample, a cross-linked PVA/GA sample, and the PVA/GA samples after treatment with freezing/thawing 1 cycle, 3 cycles, and 5 cycles (PVA/GA/FT1, PVA/GA/FT3, PVA/ GA/FT5) as presented in Fig. 2a. PVA typically forms a semicrystalline structure where the crystalline phases consist of single crystals that are bound to each other by disordered polymer chains. The freezing/thawing process resulted in the increase of crystallinity that can serve as physical cross-linkers to make it insoluble in water. It has been widely accepted that the crystallite is formed due to the folding of PVA chains via dissolution and rearrangement of PVA chains. ${ }^{17,26}$ All prepared PVA nanofibers exhibited the same reflections with an evident peak at $2 \theta \sim 19.5^{\circ}$, corresponding to the (101) reflections of polymer crystalline phases. ${ }^{27}$ This result indicates that all prepared PVA nanofibers show similar crystalline microstructures. It is worth noting that the number of freezing/thawing cycles resulted in different peak intensities, i.e., more cycles led to the increases of the intensity and the full width at half maximum of the peak; therefore, the crystallinity is controlled by the freezing/thawing process. We further analyzed the diffraction profiles to extract one of significant structural parameters for crystalline, the diameter of crystallite, to reveal the effect of the process. Since the (101) diffraction is attributed to the periodic crystal structure of PVA chains induced by the interference between the PVA chains in the direction of the intermolecular hydrogen bonding, the increase in the intensity of the (101) diffraction can be correlated to the increase in the number of well-packed regular PVA chains. The average crystallite size can be calculated from the width of the XRD peaks by Scherrer's equation given below:

$$
D=K \lambda / \beta \cos \theta
$$

where $D$ is a diameter of crystallite $(\AA), K$ is a constant $(0.89)$ taken from the literature, ${ }^{28,29} \lambda$ is a wavelength of incident X-ray, $\beta$ and $\theta$ are a full width at half maximum and a diffraction angle of the specific peak, respectively. Estimated crystallite size of pure PVA nanofiber was $23 \pm 1 \AA$; then the size gradually increased as the number of freezing/thawing process is increased (28 $\pm 2 \AA$ upon five times freezing/thawing cycles in PVA/GA/FT5) (Fig. 2b). With these results, the increase in the (101) diffraction intensity with the increasing number of freezing/thawing cycles strongly suggests that the size of the crystalline phases in PVA nanofibers can be effectively controlled by the simple process.

\section{Degree of crystallinity from FT-IR spectra}

FT-IR spectroscopy is useful to probe the crystallinity of polymers ${ }^{30,31}$ specifically the crystallinity of PVA can be easily estimated from FT-IR spectra. ${ }^{32,33}$ Yang et al. showed that the intensity ratio of the absorption band at $1142 \mathrm{~cm}^{-1}$ to the absorption band at $1095 \mathrm{~cm}^{-1}$ is strongly correlated to the crystallinity of the PVA, though the reason for the correlation is not clearly known yet; however, the bands are assigned to $\nu(\mathrm{C}-\mathrm{O})$ stretching vibration and $\nu(\mathrm{C}-\mathrm{C})$ stretching vibration in crystalline phase. ${ }^{34,35}$ It was found that the degree of crystallinity, $\chi_{\mathrm{c}}(\%)$, scales linearly to intensity ratio $\left(A_{1142} / A_{1095}\right)$ with the given empirical equation below:

$$
\chi_{\mathrm{c}}=-13.1+89.5\left(A_{1142} / A_{1095}\right)
$$

where $A_{1142}$ and $A_{1095}$ are normalized intensities at $1142 \mathrm{~cm}^{-1}$ and $1095 \mathrm{~cm}^{-1}$, respectively. Using this equation, we estimated $\chi_{c}$ from obtained FT-IR spectra of various PVA nanofiber samples. Fig. 3a displays FT-IR spectra of various PVA nanofiber samples, clearly showing that freezing/thawing process enhances the intensity ratio of the peak at 1142 and $1095 \mathrm{~cm}^{-1}$. The intensity ratio value $\left(A_{1142} / A_{1095}\right)$ extracted by further analysis varied from 0.40 (pure PVA nanofiber sample) to 0.63 (PVA/ GA/FT5), indicating the increase of the ratio by increasing the number of freezing/thawing cycles. Estimated $\chi_{\mathrm{c}}$ values of in pure PVA and PVA/GA nanofiber samples without freezing/ thawing process were found to be $23.5 \%$. However, $\chi_{\mathrm{c}}$ considerably increased up to $\sim 43.6 \%$ upon five freezing/thawing cycles. The increase of crystallinity also can be observed from the decrease of the peak at $3000-3600 \mathrm{~cm}^{-1}$, which typically
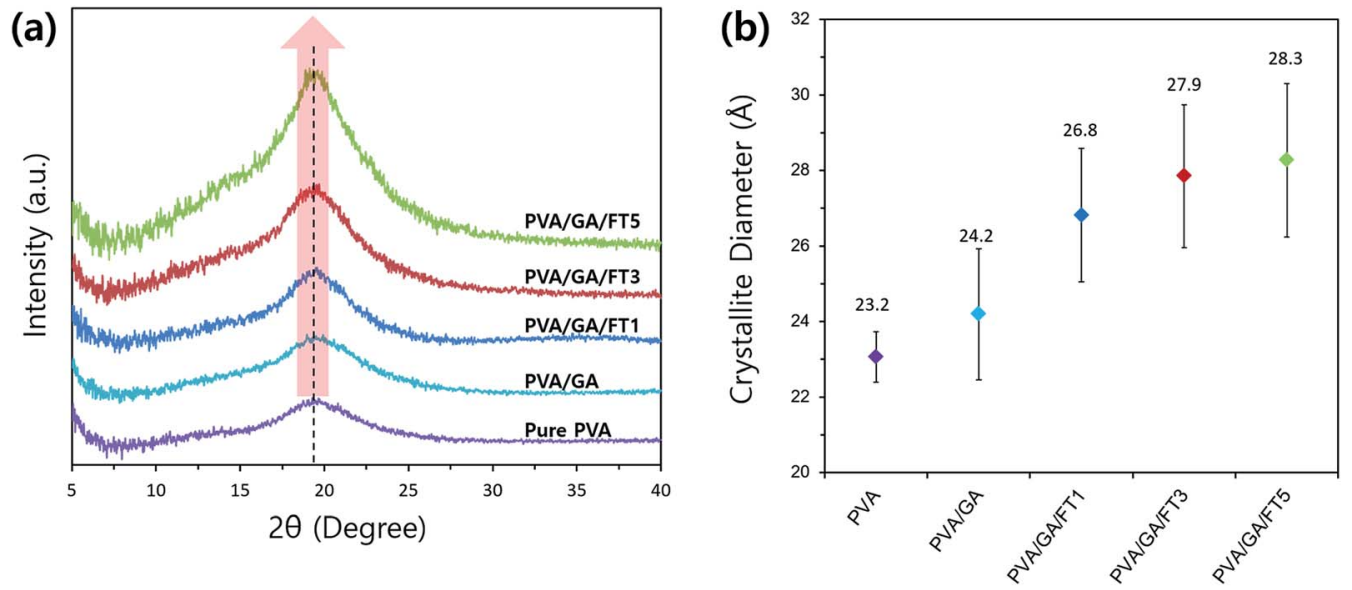

Fig. 2 (a) X-ray diffraction patterns of prepared PVA nanofibers, and (b) the calculated crystallite diameter from the pattens. 

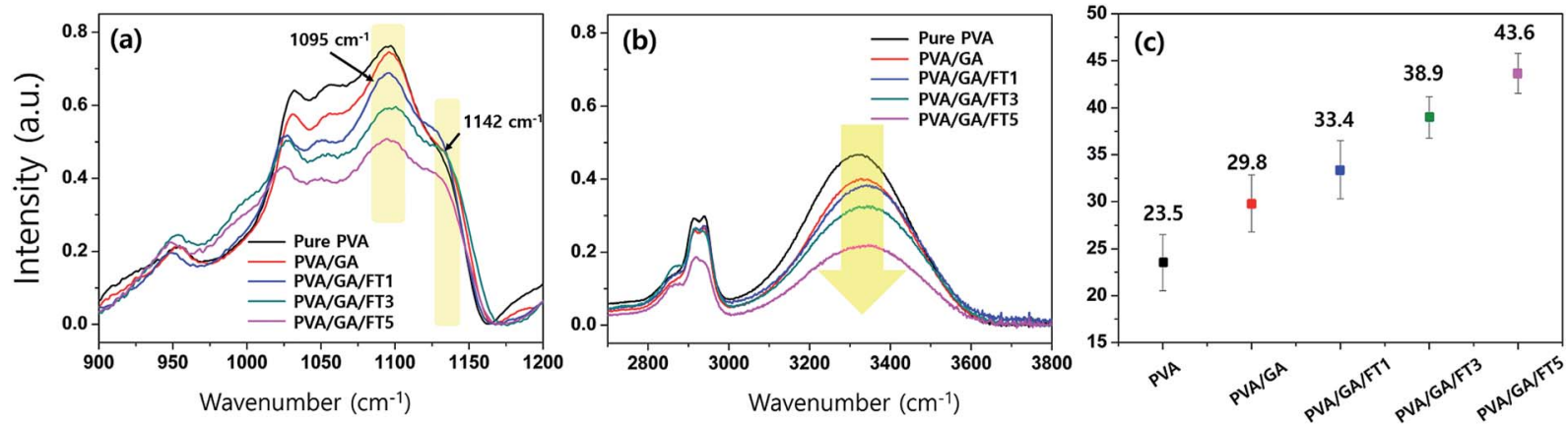

Fig. 3 ( $a$ and b) FT-IR spectra of nanofibers with different freezing/thawing cycles, and (c) the extracted degree of crystallinity from ratio of $A_{1142} /$ $A_{1095}$.

assigned to the stretching vibrations of free hydroxyl groups in PVA. A number of intrachain or interchain hydrogen bondings are formed after freezing/thawing cycles, resulting in the increase of crystallinity. By the noncovalent interaction, the amount of free hydroxyl group decreases, leading to less intense peak at $3000-3600 \mathrm{~cm}^{-1}$. In Fig. 3b, it is clear that the freezing/ thawing cycles made the intensity of the band at $3300 \mathrm{~cm}^{-1}$ considerably reduced compared to pure PVA nanofiber. Therefore, these results strongly suggest that crystallinity of PVA nanofibers can be enhanced by freezing/thawing process, ${ }^{36}$ which is also in good agreement with X-ray diffraction studies showing an effective control of crystallite size by the process.

\section{Effect of crystallinity on physical properties of nanofibers}

Crystallinity is one of important aspects that can govern physical properties such as a mechanical strength in a given material. To elucidate the effect of enhanced crystallinity to the physical properties of PVA nanofiber samples, we characterized mechanical properties of prepared nanofiber samples by measuring stress-strain curves. Fig. 4a shows representative stress-strain curves of pure PVA and cross-linked PVA nanofiber samples with varied number of freezing/thawing cycles. The PVA/GA nanofiber sample showed higher tensile strength and elongation at break $(8.2 \pm 2.2 \mathrm{MPa}$ and $84.6 \pm 10.0 \%$, respectively) than the pure PVA nanofiber sample $(4.8 \pm 2.2 \mathrm{MPa}$ and $7.2 \pm 3.0 \%$, respectively), which is expected as crosslinked network typically exhibit enhanced mechanical properties. This cross-linked structure formed by the reaction between hydroxyl group in PVA and aldehyde in GA improves the extensibility of resultant nanofiber than pure PVA nanofiber which only have semi-crystallinity, therefore the PVA/GA nanofiber sample became quite ductile. However, after freezing/thawing processes, the sample became brittle. As the sample was treated with more number of freezing/thawing cycles, the tensile strength and Young's modulus were increased and decreased from $12.6 \pm 1.3 \mathrm{MPa}$ and $34.3 \pm 10.5 \%$ ( 1 cycle) to
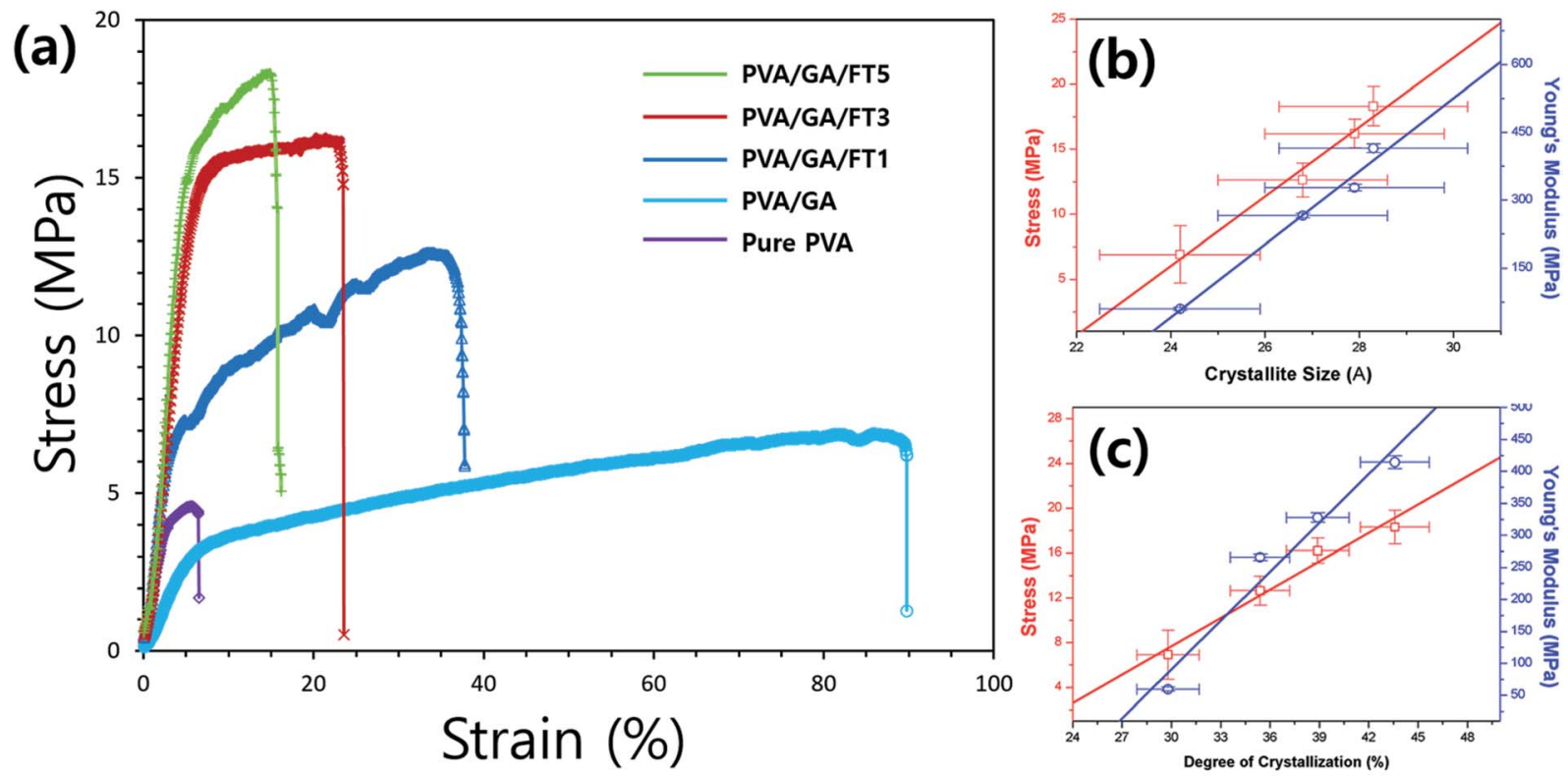

Fig. 4 (a) Stress-strain curves obtained from the samples treated with different freezing/thawing cycles, and the correlation of stress and Young's modulus to (b) crystallite size, and (c) degree of crystallinity. 
Table 2 Parameters extracted from quantitative analyses of experimental data

\begin{tabular}{lllcc}
\hline & Crystallite size $(\AA)$ & Degree of crystallinity $(\%)$ & $\sigma_{\max }(\mathrm{MPa})$ & Elongation at break $\delta(\%)$ \\
\hline PVA & $23.2 \pm 0.6$ & $23.5 \pm 1.7$ & $4.8 \pm 2.2$ & $7.2 \pm 3.0$ \\
PVA/GA & $24.2 \pm 1.7$ & $29.8 \pm 1.9$ & $8.2 \pm 2.2$ & $84.6 \pm 10.0$ \\
PVA/GA/FT1 & $26.8 \pm 1.8$ & $33.4 \pm 1.8$ & $12.6 \pm 1.3$ & $34.3 \pm 10.5$ \\
PVA/GA/FT3 & $27.9 \pm 1.9$ & $38.9 \pm 1.9$ & $15.7 \pm 1.1$ & $14.1 \pm 5.9$ \\
PVA/GA/FT5 & $28.3 \pm 2.0$ & $43.6 \pm 2.1$ & $18.3 \pm 1.5$ & $9.8 \pm 5.7$
\end{tabular}

$18.3 \pm 1.5 \mathrm{MPa}$ and $9.8 \pm 5.7 \%$ (5 cycles), respectively. The changes in tensile strength was dramatic and impactful: 5 freezing/thawing cycles made the values $\sim 165 \%$ increase compared to before the process. Fig. $4 \mathrm{~b}$ and c display the plots of tensile strength and Young's modulus as a function of crystallite diameter and degree of crystallinity, respectively, which were extracted from X-ray diffraction and FT-IR results in previous sections. Both parameters representing physical properties of the samples scale linearly to crystallite diameter $(D)$ and degree of crystallinity $\left(\chi_{\mathrm{c}}\right)$. The tensile strength and Young's modulus at $D$ of $24.2 \AA$ and $\chi_{\mathrm{c}}$ of $23.5 \%$ were 6.9 and $59.6 \mathrm{MPa}$, respectively; they increased to 18.3 and $414.2 \mathrm{MPa}$ at $D$ of $28.3 \AA$ and of $\chi_{\mathrm{c}}$ of $43.6 \%$, respectively. Therefore, the correlation suggests that the additional crystallization induced by freezing/thawing process dramatically enhances the physical strength and mechanical properties in fabricated nanofibers. Also, the linear relationship between physical properties to crystallite diameter and degree of crystallinity is worth noting. By linear regression analyses, we found four simple empirical equations: $(\mathrm{i})$ modulus $(\mathrm{MPa})=(80.9 \pm 8.5) \times D(\AA)+(-1900.5$ $\pm 229.1)$; (ii) $\sigma_{\max }(\mathrm{MPa})=(2.7 \pm 0.3) \times D(\AA)+(-58.2 \pm 7.4)$; (iii) modulus $(\mathrm{MPa})=(25.4 \pm 3.6) \times \chi_{\mathrm{c}}+(-671.9 \pm 135.8)$; (iv) $\sigma_{\max }(\mathrm{MPa})=(0.8 \pm 0.1) \times \chi_{\mathrm{c}}+(-17.7 \pm 4.0)$. These correlations strongly suggest that mechanical properties can be precisely predicted and controlled as a function of degree of crystallization or crystallite size. Therefore, these results further emphasize that this simple process can be a useful handle to achieve desirable properties in nanofibers all parameters from X-ray diffraction, FT-IR, and physical characterization in this study are summarized in Table 2.

\section{Conclusions}

We demonstrated the enhancement of physical properties in PVA nanofibers by introducing a secondary crystallization through freezing/thawing process. The structure, morphology, crystallinity (crystallite size and degree of crystallinity) and mechanical properties of PVA nanofibers upon freezing/ thawing cycles have been investigated thoroughly; the effect of the simple, facile and straightforward freezing/thawing process was impactful. First, the crystallite size of pure PVA nanofiber calculated from X-ray diffraction profile was $23.2 \AA$, which gradually increased up to $28.3 \AA$ after 5 cycles of freezing/ thawing. It was clearly observed that the intensity of (101) diffraction peak of the PVA crystal increased by the cycles. Second, the degree of crystallinity also was simultaneously increased, confirmed by close examinations of characteristic peaks in FT-IR spectra. By the quantitative analysis of the bands at $1142 \mathrm{~cm}^{-1}$ and at $1095 \mathrm{~cm}^{-1}$ and their correlation to the degree of crystallinity with the equation of $\chi_{\mathrm{c}}=-13.1+89.5$ $\left(A_{1142} / A_{1095}\right)$, it was found that $\chi_{\mathrm{c}}$ was largely increased from 23.5 to $43.6 \%$ after freezing/thawing process. The crystallinity induced by freezing/thawing process dramatically enhanced the mechanical properties of resultant PVA nanofibers compared to pristine PVA nanofibers. In addition, tensile strength and Young's modulus showed a linear relationship with degree of crystallinity and crystalline size, which will be an invaluable information for understanding and controlling PVA nanofiber property. Indeed, this simple freezing/thawing process should be beneficial for tailoring the microstructure of fibrous materials and hence, their physical and mechanical properties. Furthermore, careful characterizations with multiple tools revealed the important correlation of crystalline structure and physical properties. Our findings will open a practical route and stimulate further researches on the fibrous materials with enhanced physical properties by precisely controlling the crystallinity in the materials.

\section{Funding sources}

This research did not receive any specific grant from funding agencies in the public, commercial, or not-for-profit sectors.

\section{Conflicts of interest}

There are no conflicts to declare.

\section{Acknowledgements}

We gratefully acknowledge the support from Division of Frontier Fibers, Institute for Fiber Engineering (IFES), Interdisciplinary Cluster for Cutting Edge Research (ICCER) at Shinshu University.

\section{Notes and references}

1 Y. Liu, Y. Li and H. Xiong, ACS Macro Lett., 2013, 2, 45-48.

2 S. Cheng, D. M. Smith and C. Y. Li, Macromolecules, 2014, 47, 3978-3986.

3 O. D. Sherby and P. M. Burke, Prog. Mater. Sci., 1968, 13, 323390.

4 I. M. Ward, Proc. Phys. Soc., London, 1962, 80, 1176.

5 M. Kunioka, A. Tamaki and Y. Doi, Macromolecules, 1989, 22, 694-697. 
6 H. Lee, J. M. Koo, D. Sohn, I.-S. Kim and S. S. Im, RSC Adv., 2016, 6, 40383-40388.

7 H. Lee, K. Hun Song, S. Soon Im, J.-S. Jung, A. W. Jatoi and I. S. Kim, Nanosci. Nanotechnol. Lett., 2016, 8, 168-172.

8 H. Lee, D.-N. Phan, M. Kim, D. Sohn, S.-G. Oh, S. Kim and I. Kim, Nanomaterials, 2016, 6, 226.

9 Z.-M. Huang, Y. Z. Zhang, M. Kotaki and S. Ramakrishna, Compos. Sci. Technol., 2003, 63, 2223-2253.

10 M. Dhanalakshmi and J. Jog, eXPRESS Polym. Lett., 2008, 2, 540-545.

11 X. Wang, H. Zhao, L.-S. Turng and Q. Li, Ind. Eng. Chem. Res., 2013, 52, 4939-4949.

12 J. Junkasem, R. Rujiravanit, B. P. Grady and P. Supaphol, Polym. Int., 2010, 59, 85-91.

13 D. Cao, Z. Fu and C. Li, Mater. Sci. Eng., B, 2011, 176, 900905.

14 R. Ricciardi, F. Auriemma, C. De Rosa and F. Lauprêtre, Macromolecules, 2004, 37, 1921-1927.

15 C. M. Hassan and N. A. Peppas, Macromolecules, 2000, 33, 2472-2479.

16 H. Zhang, H. Xia and Y. Zhao, ACS Macro Lett., 2012, 1, 12331236.

17 N. A. Peppas and S. R. Stauffer, J. Controlled Release, 1991, 16, 305-310.

18 T. Fukumori and T. Nakaoki, Open J. Org. Polym. Mater., 2013, 3, 110.

19 C. C. DeMerlis and D. R. Schoneker, Food Chem. Toxicol., 2003, 41, 319-326.

20 H. Lee, M. Kim, D. Sohn, S. H. Kim, S.-G. Oh, S. S. Im and I. S. Kim, RSC Adv., 2017, 7, 6108-6113.

21 H. Lee, K. Watanabe, M. Kim, M. Gopiraman, K.-H. Song, J. S. Lee and I. S. Kim, Sci. Rep., 2016, 6, 37590.
22 J. L. Koenig, in Spectroscopy: NMR, Fluorescence, FT-IR, Springer Berlin Heidelberg, Berlin, Heidelberg, 1984, pp. 87-154, DOI: 10.1007/3-540-12591-4_6.

23 O. N. Tretinnikov and S. A. Zagorskaya, J. Appl. Spectrosc., 2012, 79, 521-526.

24 K.-O. Kim, Y. Akada, W. Kai, B.-S. Kim and I.-S. Kim, J. Biomater. Nanobiotechnol., 2011, 2, 8.

25 C.-K. Yeom and K.-H. Lee, J. Membr. Sci., 1996, 109, 257-265.

26 S. R. Stauffer and N. A. Peppast, Polymer, 1992, 33, 39323936.

27 Y. Nishio and R. J. Manley, Macromolecules, 1988, 21, 12701277.

28 P. Sivakumar, R. Ramesh, A. Ramanand, S. Ponnusamy and C. Muthamizhchelvan, Mater. Res. Bull., 2011, 46, 22082211.

29 Z. Y. Li, W. M. Lam, C. Yang, B. Xu, G. X. Ni, S. A. Abbah, K. M. C. Cheung, K. D. K. Luk and W. W. Lu, Biomaterials, 2007, 28, 1452-1460.

30 S. K. Mallapragada and N. A. Peppas, J. Polym. Sci., Part B: Polym. Phys., 1996, 34, 1339-1346.

31 X.-D. Ma, X.-F. Qian, J. Yin and Z.-K. Zhu, J. Mater. Chem., 2002, 12, 663-666.

32 X. Yang, Z. Zhu, Q. Liu and X. Chen, J. Appl. Polym. Sci., 2008, 109, 3825-3830.

33 K. Sugiura, M. Hashimoto, S. Matsuzawa and K. Yamaura, J. Appl. Polym. Sci., 2001, 82, 1291-1298.

34 O. N. Tretinnikov and S. A. Zagorskaya, J. Appl. Spectrosc., 2012, 79, 521-526.

35 Y.-L. Wang, H. Yang and Z.-L. Xu, J. Appl. Polym. Sci., 2008, 107, 1423-1429.

36 H. S. Mansur, C. M. Sadahira, A. N. Souza and A. A. P. Mansur, Mater. Sci. Eng., C, 2008, 28, 539-548. 